\title{
Plasma Neutrophil Gelatinase-Associated Lipocalin and Interleukin-9 as Acute Kidney Injury Predictors After Coronary Artery Bypass Graft Following Cardiopulmonary Bypass
}

\author{
Ghuraba Adisurya, Kun Arifi Abbas*, \\ Nancy Margarita Rehatta and Hardiono
}

\author{
Anesthesiology and Intensive Care Department, Faculty of Medicine \\ Universitas Airlangga, Surabaya 60286 Indonesia \\ *Corresponding author details: Kun Arifi Abbas; kunarifi@gmail.com
}

\begin{abstract}
Background: Acute Kidney Injury (AKI) incidence of postoperative Coronary Artery Bypass Graft (CABG) with Cardipulmonary Bypass (CPB) at Dr. Soetomo General Academic Hospital reached 69.8\%, higher than the global average of 5-20\%. Predictors of postoperative AKI are needed; potential biomarkers include plasma Neutrophil Gelatinase-Associated Lipocalin (NGAL) and Interleukin-9 (IL-9). This study aims to analyze the predictive ability of these two biomarkers.

Methods: This is a prospective observational study in CABG patients with CPB without previous renal abnormalities at Dr. Soetomo in September-December 2020. Examination of plasma NGAL and IL-9 was carried out before, during, and after the CPB procedure.

Results: 21 patients were included in the study, 17 people $(81.0 \%)$ were male. AKI experienced 14 people $(66.7 \%) ; 3$ people $(14.3 \%)$ died. Patients without AKI had higher plasma NGAL results at preinduction $(17.9 \pm 10.3$ vs $17.1 \pm 15.5)$, durante $(20.5 \pm 8.2$ vs $16.9 \pm 7.8)$, and early ICU $(24.0 \pm 8.6$ vs $22.9 \pm 10.4)$. Patients with AKI had higher plasma IL-9 results at preinduction (63.3 \pm 23.2 vs $55.7 \pm 15.8$ ), durante (45.2 \pm 11.0 vs $44.2 \pm 9.2$ ), and early ICU $(49.6 \pm 11.3$ vs $46.2 \pm 15.6)$. There was no statistically significant relationship between plasma NGAL and IL- 9 results.

Conclusion: Most CABG patients undergoing CPB have AKI. Patients without AKI had higher NGAL results and lower IL-9 in plasma. Statistically, neither of them can be used as predictive biomarkers of the incidence of AKI so far.
\end{abstract}

Keywords: NGAL; IL-9; Plasma; AKI; CPB; CABG

\section{INTRODUCTION}

The Coronary Artery Bypass Graft (CABG) procedure with the Cardiopulmonary Bypass (CPB) technique has a major drawback, namely systemic ischemia in which one of the target organs is the kidney(Setiari et al., 2017). The presence of hypoperfusion, ischemic-reperfusion injury, neurohumoral activation, activation of the inflammatory response, oxidative stress, nephrotoxins, coagulation disorders, and mechanical factors lead to decreased kidney function and eventually Acute Kidney Injury (AKI) occurs (Prabhu et al., 2010); (Wang \& Bellomo, 2017). There are several conditions associated with the incidence of postoperative AKI CABG with $\mathrm{CPB}$, namely old age and female gender, preoperative cardiac dysfunction, emergency surgery, peripheral arterial disease, reintervention, diabetes requiring insulin, intraoperative use of aprotinin, suffering from obstructive pulmonary disease. chronic obstructive pulmonary disease (COPD), and preoperative renal dysfunction (Vives et al., 2019).

In the United States, the number of CABG operations is estimated at 200 thousand per year (Melly et al., 2018). Meanwhile in Indonesia, there is no national data that conveys the epidemiological data yet. However, it is known that at Dr. Soetomo General Academic Hospital Surabaya, Indonesia, the CABG procedure in 2018 and 2019 has reached 82 and 64 times. It is estimated that about 5-20\% of CABG operations using a CPB machine will have postoperative AKI complications and this will increase the risk of mortality by $>60 \%$ (Prabhu et al., 2010). Searching in our preliminary study about the medical records of Dr. Soetomo General Academic Hospital Surabaya, Indonesia, showed that the incidence of AKI after CABG surgery using a CPB machine in 2019 reached 63.8\%. Thus, the identification of renal dysfunction before surgery is important to predict the occurrence of AKI after surgery (Wang \& Bellomo, 2017).

Neutrophil gelatinase-associated lipocalin is a potent renal troponin consisting of eight $b$-strains forming a b-barrel calyx for binding and transporting low-molecular chemicals (Soni et al., 2010). NGAL levels are known to increase early in the course of AKI. This was conveyed by a review study in 2017 which stated that the use of NGAL combined with other kidney function assessments could be useful for early detection of AKI related to heart surgery(Vandenberghe et al., 2017). Biomarker markers have a high predictor value in post-cardiac surgery AKI and can be used for early detection where it will appear in plasma within hours after kidney damage occurs so it is more sensitive than serum creatinine and urine production (O'Neal JB, et al. 2016; Fadel FI, et al. 2012; Antonucci E, et al. 2014; Mosa OF. 2018). 
Another biomarker that is also thought to be used to predict the occurrence of AKI is interleukin-9 (IL-9). Interleukin-9 is a pleiotropic inflammatory cytokine produced by various inflammatory cells, such as mast cells, natural killer cells, T-helper cells, T-regulatory cells, and others. These cytokines primarily function as regulators of the activity of hematopoietic cells(Noelle \& Nowak, 2010). IL-9 levels are known to increase during monocyte activation due to the inflammatory response by TNF- and IL-18 in a cohort study showing that IL-9 levels can be used to differentiate the diagnosis of acute interstitial nephritis from the diagnosis of acute kidney injury(Moledina \& Parikh, 2019).With this description, this study was designed to analyze levels of neutrophil gelatinaseassociated lipocalin and plasma interleukin-9 which were used as predictors of the incidence of AKI after coronary artery bypass graft surgery with cardiopulmonary bypass.

\section{METHOD}

This study is an observational analytic study with a prospective longitudinal design used to analyze the role of plasma NGAL and IL-9 levels as predictors of AKI after CABG surgery with $\mathrm{CPB}$. Prospective longitudinal means that this study takes data over a certain period of time, over a long period of time, on future findings.

The research begins with the submission of research proposals, ethical management, and licensing arrangements at the research location. After all, three are completed, the research continues with the selection of samples from the population. Then, IL-9 and NGAL were examined on all research samples. Researchers then recorded the incidence of AKI. The study concludes with a presentation, evaluation, and publication.

The sample of this study were patients who underwent CABG surgery with the CPB technique at Dr. SoetomoGeneral Academic Hospital Surabaya, Indonesia, during SeptemberDecember 2020 who met the inclusion and exclusion criteria This research was conducted at the Central Surgical Installation, Integrated Cardiac Service Center, nursing ward, and ICU Dr. Soetomo General Academic Hospital Surabaya, Indonesia, during September-December 2020.

\section{RESULT}

This research was conducted in Dr. Soetomo General Academic Hospital Surabaya, Indonesia, A total of 21 patients were included in this study. The inclusion criteria of this study were all patients undergoing CPB at Dr. Soetomo General Academic Hospital Surabaya, Indonesia, with complete examination data.

The sample of this study was dominated by men with a total of $17(81.0 \%)$. The most common comorbidities were hypertension and a combination of hypertension and diabetes mellitus, namely 4 samples (19\%) and uncontrolled comorbidities with $18(85.7 \%)$. The majority of patients undergoing CPB had AKI with a total of 15 (71.4\%). Outcome ad vitam obtained as many as 3 samples $(14.3 \%)$ with the survivors as many as 18 samples (85.7\%). Most of the KRS samples on day 5 and 7 were 5 samples (23.8\%) followed by day 6 and 3 samples died (14.3\%).

The basic characteristics of the study using a numerical scale where the mean \pm SD of the patient's age was $58.7 \pm$ 8.6 years, the patient's BMI was $24.5 \pm 3.0$, the length of ICU stay was $2.6 \pm 1.0$ days, the length of action was $413.8 \pm$ 72.2 minutes, ejection fraction (\%) $58 \pm 9.69$, and total post ICU $3.64 \pm 1.05$.

The results of the Shapiro-Wilk test showed that only NGAL and IL-9 pre CPB data in the AKI group and early ICU IL-9 data in the group without AKI were not normally

distributed ( $\mathrm{p}<0.05)$, while the NGAL and IL-9 data during $\mathrm{CPB}$ and Other early ICUs were normally distributed. Differences in NGAL and IL-9 pre CPB and IL-9 early ICU between the AKI and without AKI groups were analyzed using the Mann Whitney test, while the differences in NGAL and IL-9 during CPB and early ICU NGAL between the AKI and without AKI groups were analyzed using the T test. 2 free samples.

TABLE 1: The test results of the normal distribution of NGAL and IL-9 deltas data

\begin{tabular}{|l|c|c|}
\hline \multirow{2}{*}{ Biomarker } & \multicolumn{2}{|c|}{ P Value } \\
\cline { 2 - 3 } & AKI (n = 14) & Non-AKI (n = 7) \\
\hline NGAL & & 0,582 \\
\hline Pre CPB & 0,003 & 0,288 \\
\hline Durante CPB & 0,389 & 0,178 \\
\hline Early ICU & 0,445 & \\
\hline IL-9 & & 0,680 \\
\hline Pre CPB & 0,027 & 0,339 \\
\hline Durante CPB & 0,990 & 0,019 \\
\hline Early ICU & 0,311 & \\
\hline
\end{tabular}

Based on Table 1, the results of the Shapiro-Wilk test show that only NGAL and IL-9 pre CPB data in the AKI group and early ICU IL-9 data in the group without AKI were not normally distributed $(\mathrm{p}<0.05)$. Meanwhile, data on NGAL and IL-9 during CPB and other Early ICUs were normally distributed. Differences in NGAL and IL-9 pre CPB and IL-9 early ICU between the AKI and without AKI groups were analyzed using the Mann Whitney test, while the differences in NGAL and IL-9 during CPB and early ICU NGAL between the AKI and without AKI groups were analyzed using the T test. 2 free samples.

\section{Effect of NGAL on the incidence of AKI after CPB}

Table 2 below is compiled to show the analysis of the relationship between Neutrophil Gelatinase-Associated Lipocalin and the incidence of AKI in patients with CPB. 
TABLE 2: The relationship between Neutrophil Gelatinase-Associated Lipocalin and the incidence of AKI.

\begin{tabular}{|l|c|c|c|}
\cline { 2 - 4 } \multicolumn{1}{c|}{} & \multicolumn{3}{c|}{ AKI Incidence (Mean \pm SD) } \\
\cline { 2 - 4 } \multicolumn{1}{c|}{} & Yes & No & p-value \\
\hline NGAL Preinduction & $17,1 \pm 15,5$ & $17,9 \pm 10,3$ & $0,900^{*}$ \\
\hline NGAL Durante & $16,9 \pm 7,8$ & $20,5 \pm 8,2$ & $0,343^{+}$ \\
\hline NGAL Early ICU & $22,9 \pm 10,4$ & $24,0 \pm 8,6$ & $0,816^{+}$ \\
\hline
\end{tabular}

*: Mann Whitney, +: T-Test

Table 2 shows that patients without AKI had higher NGAL than those with AKI both at preinduction $(17.9 \pm 10.3$ vs. 17.1 $\pm 15.5)$, durante $(20.5 \pm 8.2$ vs. $16.9 \pm 7.8)$ and early ICU $(24.0$ \pm 8.6 vs. $22.9 \pm 10.4$ ). There was no statistically significant relationship between preinduction NGAL (p-value 0.900), durante (p-value 0.343 ) and early ICU (p-value 0.816).

\section{Effect of IL-9 on the Incidence of Post-CPB AKI}

Table 3 below shows an analysis of the relationship between IL- 9 and the incidence of AKI in patients with CPB.

TABLE 3: The relationship between IL-9 and the incidence of AKI

\begin{tabular}{|lc|c|c|}
\cline { 2 - 4 } \multicolumn{1}{c|}{} & \multicolumn{3}{c|}{ AKI Incident (Mean \pm SD) } \\
\cline { 2 - 4 } \multicolumn{1}{c|}{} & Yes & No & p-value \\
\hline IL-9 Preinduction & $63,3 \pm 23,2$ & $55,7 \pm 15.8$ & $0,444^{*}$ \\
\hline IL-9 Durante & $45,2 \pm 11.0$ & $44,2 \pm 9,2$ & $0,838^{+}$ \\
\hline IL-9 Early ICU & $49,6 \pm 11,3$ & $46,2 \pm 15,6$ & $0,351^{*}$ \\
\hline
\end{tabular}

*: Mann Whitney, +: T-Test

Table 3 shows that patients with AKI had higher IL-9 than those without AKI either at preinduction $(63.3 \pm 23.2$ vs. $55.7 \pm 15.8$ ), durante ( $45.2 \pm 11.0$ vs. $44,2 \pm 9.2$ ) and early ICU ( $49.6 \pm 11.3$ vs. $46.2 \pm 15.6)$. There was no statistically significant relationship between IL-9 preinduction ( $\mathrm{p}$ value 0.444 ), durante ( $\mathrm{p}$-value 0.838 ), and early ICU ( $\mathrm{p}$ value 0.351 ).

\section{Effect of Delta NGAL with Post-CPB AKI Incidence}

Table 4 below shows an analysis of the relationship between delta NGAL and the incidence of AKI in patients with CPB.

TABLE 4: The relationship between the NGAL delta and the incidence of AKI

\begin{tabular}{|l|c|c|c|}
\cline { 2 - 4 } \multicolumn{1}{c|}{} & \multicolumn{2}{c|}{ AKI Incident (Mean \pm SD) } \\
\cline { 2 - 4 } \multicolumn{1}{c|}{} & Yes & No & p-value \\
\hline Delta NGAL Durante- Preinduction & $-0,2 \pm 16,0$ & $2,6 \pm 7,4$ & $0,657^{*}$ \\
\hline Delta NGAL Early ICU-Preinduction & $5,9 \pm 15,3$ & $6,1 \pm 11,0$ & $0,968^{+}$ \\
\hline Delta NGAL Early ICU-Durante & $6,0 \pm 10,6$ & $3,5 \pm 5,3$ & $0,546^{+}$ \\
\hline
\end{tabular}

*: Mann Whitney, +: T-Test

Table 4 shows that patients without AKI had higher NGAL deltas than those with AKI during durante-preinduction $(2.6$ \pm 7.4 vs $-0.2 \pm 16.0$ ) and early ICU-preinduction $(6,1 \pm 11.0$ vs. $5.9 \pm 15.3$ ), while in early ICU-durante it was higher in those with AKI than those without ( $3.5 \pm 5.3$ vs. $6.0 \pm 10.6)$. Statistically significant relationships were not found in the NGAL delta durante-preinduction (p-value 0.657), early ICU-preinduction (p-value 0.968) and early ICUdurante (p-value 0.546).

\section{Effect of Delta IL-9 with Post-CPB A AKI Incidence}

Table 5 below shows an analysis of the relationship between delta IL-9 and the incidence of AKI in patients with CPB.

TABLE 5: Relationship between delta IL-9 and the incidence of AKI

\begin{tabular}{|c|c|c|c|}
\cline { 2 - 4 } \multicolumn{1}{c|}{} & \multicolumn{2}{c|}{ AKI Incident (Mean \pm SD) } \\
\cline { 2 - 4 } \multicolumn{1}{c|}{} & Yes & No & p-value \\
\hline Delta IL-9 Durante-Preinduction & $-18,1 \pm 25,8$ & $-11,5 \pm 15,8$ & $0,518^{*}$ \\
\hline Delta IL-9 Early ICU-Preinduction & $-13,7 \pm 19,7$ & $-9,4 \pm 19,0$ & $0,622^{+}$ \\
\hline Delta IL-9 Early ICU-Durante & $4,4 \pm 11,9$ & $2,05 \pm 16,7$ & $0,692^{+}$ \\
\hline
\end{tabular}

*: Mann Whitney, +: T-Test 
Table 5 shows that patients with AKI had higher IL-9 deltas than those without AKI during durante-preinduction ($18.1 \pm 25.8$ vs $-11.5 \pm 15.8)$, early ICU-preinduction $(-13.7$ \pm 19.7 vs $-9.4 \pm 19.0$ ) and early ICU-durante ( $4.4 \pm 11.9$ vs. $2.05 \pm 16.7$ ). Statistically significant relationship was not seen in delta IL-9 durante-preinduction ( $p$-value 0.518), early ICU-preinduction ( $p$-value 0.622), and early ICUdurante (p-value 0.692).

\section{DISCUSSION}

\section{Characteristic}

Acute kidney injury (AKI) covers a broad spectrum of kidney damage and often causes acute renal failure (ARF) which is a rapid and sustained decline in kidney function. According to the Acute Kidney Injury Network (AKIN) criteria, AKI usually causes an increase in serum creatinine (SCr) of more than 50\%. Despite improvements in clinical care, AKI remains a serious problem with an increasing incidence(Kasper; Denis L; et al, 2018).

The clinical manifestations of AKI vary from a minimal but sustained decrease in the glomerular filtration rate (GFR) to auric renal failure. Acute kidney injury can be associated with metabolic conditions such as metabolic acidosis, hyperkalemia, and changes in body fluid balance depending on the severity and duration of the disease(Antonucci et al., 2014; Eknoyan \& Lameire, 2013).

The study sample was dominated by men with a total of 17 $(81.0 \%)$ study samples with uncontrolled comorbidities with a total of $18(85.7 \%)$ study samples. More men experienced AKI (12 samples), than women (3 samples) Those with comorbidities were controlled (14 samples) than not (1 sample). Another study involving 401 patients showed that $56.6 \%$ of the men in their study group had AKI (Tejera D et al., 2017).

Lagny's study which also examined the incidence of AKI in patients who had undergone cardiopulmonary surgery showed that male patients were more dominant (70.0\%) experienced AKI. The mean age is older, which is 69 years Preoperative hypertension diagnosis was found in $81.0 \%$ of patients. Most of the patients $(76.7 \%)$ had normal renal function before surgery. Patients with preoperative CKD are significantly older than individuals with normal renal function (Lagny MG et al., 2015).

The majority of patients undergoing CPB had AKI with a total of $14(66.7 \%)$ study samples. The incidence rate of CPB-associated AKI in Pickering's study was $18.2 \%$, and $2.1 \%$ of those requiring renal replacement therapy because CPB-associated AKI was associated with premature death (risk ratio [RR], 4.0; 95\% CI, 3.1-5.2 ; crude mortality with CPB-associated AKI, $4.6 \%$; without CPB-AKI, 1.5\%) with considerable heterogeneity between studies(Pickering et al., 2015).

The mean age of the patients in this study was $58.7 \pm 8.6$ years. The patient's Body Mass Index (BMI) was $24.5 \pm 3.0$, the length of stay in the ICU was $2.6 \pm 1.0$ days, and the length of action was $413.8 \pm 72.2$ minutes. Acute kidney injury occurs in approximately $5 \%$ of hospitalized patients and in more than $30 \%$ of patients in the intensive care unit (ICU) and is associated with high rates of morbidity and mortality. Despite advances in cardiac surgical technique and technology, AKI continues to be an important complication also in this setting(Antonucci et al., 2014; Eknoyan \& Lameire, 2013).

Relationship between NGAL and AKI in CPB Patients Acute kidney injury (AKI) is estimated to occur in $5 \%$ of hospitalized patients and as many as two thirds of intensive care unit patients. Early detection of AKI can facilitate timely intervention before the injury becomes irreversible in order to limit morbidity and mortality. The diagnosis of AKI is based on changes in serum creatinine, which may not manifest for several hours after the initial disturbance(Gabbard W et al., 2010; Kasper; Denis L; et al, 2018).

Neutrophil Gelatinase-Associated Lipocalin is a small (i.e., $25 \mathrm{kDa}$ ) protein that is part of the lipocalin family, originally discovered in 2003 by genome analysis of kidney genes induced in response to experimental AKI in animals. Neutrophil Gelatinase-Associated Lipocalin is primarily expressed by neutrophils and proximal renal tubules, while it can also be produced at much lower levels in prostate and respiratory and gastrointestinal epithelium (Dent CL et al., 2007).

Serum creatinine is an inadequate marker for early detection of AKI. First, more than $50 \%$ of renal function must be lost before an increase in serum creatinine is detected. Second, serum creatinine does not accurately reflect renal function until a steady state is reached and it may take several days after kidney damage. Animal studies have shown that AKI can be prevented and/or treated using several maneuvers, this should be done very early after kidney damage and long before an increase in serum creatinine is apparent. Monitoring of plasma NGAL levels has the potential to provide early warning to critical care providers (Dent CL et al., 2007).

Neutrophil Gelatinase-Associated Lipocalin can already be used as a stress marker because it is widely expressed in cells after infection, inflammation, ischemia, or neoplastic transformation. Its small size and resistance to degradation make NGAL easily detectable in both blood and urine. The potential use of NGAL as a biomarker of AKI has been recognized because it is one of the earliest and most rapidly induced genes in the kidney after ischemia or nephrotoxicity. injury to animal models(Antonucci E et al., 2014).

This study showed that patients without AKI had higher NGAL than those with AKI both at preinduction (17.9 \pm 10.3 vs. $17.1 \pm 15.5)$, durante $(20.5 \pm 8.2$ vs. $16.9 \pm 7.8)$ and early ICU $(24.0 \pm 8.6$ vs. $22.9 \pm 10.4)$. There was no statistically significant relationship between preinduction NGAL (p-value 0.900), durante (p-value 0.343) and early ICU (p-value 0.816). Meanwhile, in the calculation of changes or NGAL deltas, patients who did not experience AKI were higher than those with AKI in Pre $(3.5 \pm 7.6$ vs $0.3 \pm 15.4$ ) and durante ( $7.6 \pm 11.2$ vs. $5.3 \pm 14.9$ ), whereas in the early ICU it was higher in those with AKI than those without ( $5.6 \pm 10.3$ vs. $4.0 \pm 5.6)$. Statistically significant relationships were not found in the NGAL delta durantepreinduction ( $p$-value 0.657 ), early ICU-preinduction ( $p$ value 0.968 ) and early ICU-durante (p-value 0.546 ).

Postoperative Neutrophil Gelatinase-Associated Lipocalin best identifies AKI in patients with a glomerular filtration rate (GFR) of 90 to $120 \mathrm{ml} / \mathrm{min}$. Patients with baseline GFR $<60 \mathrm{ml} / \mathrm{min}$ generally have a urinary NGAL that does not differ between those with and without AKI(Bennett $M$ et al., 2008).

Renal impairment during extracorporeal circulation can be caused by decreased renal perfusion, so that an increase in NGAL expression is expected to occur in the intraoperative period or during surgery in this study. The significant decrease in uNGAL at baseline CPB suggests that the condition may be due to hemodilution at induction of anesthesia. After 2 hours of using CPB and at the end of the operation and after that a significant improvement of uNGAL was seen(Friedrich MG et al., 2017). 
Relationship between IL-9 and AKI in CPB Patients Inflammation is a rapid, intense, and non-specific systemic reaction to tissue injury involving cellular and humoral mechanisms. During cardiac surgery, a strong general inflammatory response is activated by surgical trauma, blood loss, transfusion, and temperature changes. This response has a significant clinical impact. Inflammatory changes in the outcome of the procedure are high priority targets for the development of treatment and prevention strategies (Aljure OD \& Fabbro M, 2019).

Changes in the time course, magnitude, or pattern of cytokine release after $\mathrm{CPB}$ may contribute to abnormalities in the inflammatory response to cardiac surgery. These patterns are important to understand if yield metrics are to be improved. During CPB, several inflammatory mechanisms are triggered. Initially, contact of blood with foreign surfaces of the $\mathrm{CPB}$ circuit activates the coagulation cascade and the complement system. Complement factors and their degradation products can exert immunomodulatory effects, induce the synthesis of proinflammatory cytokines, strengthen systemic responses respons (Aljure OD \& Fabbro M, 2019).

The IL-9 gene is another inflammatory mediator located on chromosome 5. IL-9 was first described as a growth factor secreted by Th2 cells. Th17, regulatory T cells (Treg), TGFb, and IL-4 also increase IL-9 secretion. IL-9 has various functions in immune and inflammatory responses by passing through the $\mathrm{C}$ family of receptors on target cells. These cytokines stimulate cell differentiation and prevent apoptosis (Jouybar R et al., 2020).

IL-9 can act as a positive and negative regulator of the immune response. In other words, IL-9 has different effects on disease progression. Melatonin is an N-acetyl-5methoxy-tryptamine chemical that is secreted by tryptophan from the pineal gland into the bloodstream and cerebrospinal fluid. Melatonin plays an important role in regulating the biological clock in humans and has a wide range of antioxidant properties. Melatonin antioxidant mechanisms are directly (through free radical scavenging) or indirectly (by regulating the activity of antioxidant enzymes) involved. Melatonin reduces surgical-related oxidative damage and ischemic reperfusion injury cedera (Jouybar R et al., 2020).

A wide spectrum of immunological functions have been associated with Interleukin 9 (IL-9), including effects on the survival and proliferation of immune cells and parenchyma. Evidence suggests that IL-9 expression can promote tissue repair under inflammatory conditions. However, data on the involvement of IL-9 in renal tissue protection are limited. Xiong (2020) investigated the role of IL-9 in the occurrence of nephropathy in a mouse model (Xiong T et al., 2020).

This study showed that patients with AKI had higher IL-9 than those without AKI both at preinduction $(63.3 \pm 23.2$ vs. $55.7 \pm 15.8)$, durante $(45.2 \pm 11.0$ vs. $44.2 \pm 9.2)$ and early ICU $(49.6 \pm 11.3$ vs. $46.2 \pm 15.6)$. There was no statistically significant relationship between IL-9 preinduction ( $p$-value 0.444$)$, durante ( $p$-value 0.838$)$, and early ICU (p-value 0.351). Likewise, in the analysis of changes or deltas of IL-9 in patients with AKI had higher IL-9 deltas than those without AKI during durantepreinduction $(-18.1 \pm 25.8$ vs $-11.5 \pm 15.8)$, early ICUpreinduction $(-13.7 \pm 19.7$ vs $-9.4 \pm 19.0)$ and early ICUdurante $(4.4 \pm 11.9$ vs. $2.05 \pm 16.7)$. Statistically significant relationship was not seen in delta IL-9 durantepreinduction ( $\mathrm{p}$-value 0.518 ), early ICU-preinduction ( $\mathrm{p}$ value 0.622 ), and early ICU-durante ( $p$-value 0.692 ).

\section{CONCLUSION}

From this study it can be concluded that most CABG patients undergoing CPB have AKI. Patients without AKI had higher NGAL results and lower plasma IL-9. Therefore, statistically both cannot be used as predictive biomarkers of the incidence of AKI.

\section{REFERENCES}

[1] Aljure OD, \& Fabbro M. (2019). Cardiopulmonary Bypass and Inflammation: The Hidden Enemy. Journal of Cardiothoracic and Vascular Anesthesia, 33, 346-347.

[2] Antonucci, E., Lippi, G., \& Ticinesi, A. (2014). Neutrophil gelatinase-associated lipocalin (NGAL): a promising biomarker for the early diagnosis of acute kidney injury (AKI). Acta Biomed, 85(1), 289-294.

[3] Antonucci E, Lippi G, Ticinesi A, \& et al. (2014). Neutrophil gelatinase-associated lipocalin (NGAL): a promising biomarker for the early diagnosis of acute kidney injury (AKI). Acta Biomed, 85(1), 289-294.

[4] Bennett M, Dent CL, Ma Q, \& et al. (2008). Urine NGAL predicts severity of acute kidney injury after cardiac surgery: a prospective study. Clin J Am Soc Nephrol, 3, $665-673$.

[5] Dent CL, Ma Q, Dastrala S, \& et al. (2007). Plasma neutrophil gelatinase-associated lipocalin predicts acute kidney injury, morbidity and mortality after pediatric cardiac surgery: a prospective uncontrolled cohort study. Crit Care, 11(6), 1-8.

[6] Eknoyan, G., \& Lameire, N. (2013). KDIGO 2012 Clinical Practice Guideline for the Evaluation and Management of Chronic Kidney Disease. In National Kidney Foundation (Vol. 3, Issue 1). https://doi.org/10.3182/20140824-6-za1003.01333

[7] Friedrich MG, Bougioukas I, Kolle J, \& et al. (2017). NGAL expression during cardiopulmonary bypass does not predict severity of postoperative acute kidney injury. BMC Nephrol, 18, 73-79.

[8] Gabbard W, Milbrandt EB, \& Kellum JA. (2010). NGAL: an emerging tool for predicting severity of AKI is easily detected by a clinical assay. Crit Care, 14(4), 318.

[9] Jouybar R, Setoodeh M, Saravi ZF, \& et al. (2020). The Effect of Melatonin on the Serum Level of Interleukin 6 and Interleukin 9 in Coronary Artery Bypass Grafting Surgery. Asian Journal of Anesthesiology, 58, $35-44$.

[10] Kasper; Denis L; et al. (2018). Harrison's Principles of Internal Medicine 19th Edition. McGraw-Hill Education.

[11] Lagny MG, Jouret F, Koch JN, \& et al. (2015). Incidence and outcomes of acute kidney injury after cardiac surgery using either criteria of the RIFLE classification. BMC Nephrology, 16(76), 59-67.

[12] Melly, L., Torregrossa, G., Lee, T., Jansens, J. L., \& Puskas, J. D. (2018). Fifty years of coronary artery bypass grafting. Journal of Thoracic Disease, 10(3), 1960-1967.

https://doi.org/10.21037/jtd.2018.02.43 
[13] Moledina, D. G., \& Parikh, C. R. (2019). Differentiating Acute Interstitial Nephritis from Acute Tubular Injury: A Challenge for Clinicians. Nephron, 143(3), 211-216. https://doi.org/10.1159/000501207

[14] Noelle, R. J., \& Nowak, E. C. (2010). Cellular sources and immune functions of interleukin-9. Nature Reviews Immunology, 10(10), 683-687. https://doi.org/10.1038/nri2848

[15] Pickering, J., James, M., \& Palmer, S. (2015). Acute kidney injury and prognosis after cardiopulmonary bypass: a meta-analysis of cohort studies. Am J Kidney Dis, 65(2), 283-293.

[16] Prabhu, A., Sujatha, D. I., Ninan, B., \& Vijayalakshmi, M. A. (2010). Neutrophil Gelatinase Associated Lipocalin as a Biomarker for Acute Kidney Injury in Patients Undergoing Coronary Artery Bypass Grafting with Cardiopulmonary Bypass. Annals of Vascular Surgery, 24(4), 525-531. https://doi.org/10.1016/j.avsg.2010.01.001

[17] Setiari, T. D., Sudjud, R. W., \& Redjeki, I. S. (2017). Korelasi antara Lama Pintas Jantung Paru dan Lama Bantuan Ventilasi Mekanis pada Pasien Pascabedah Pintas Arteri Koroner di Unit Perawatan Intensif Jantung Rumah Sakit Dr. Hasan Sadikin Bandung. Jurnal Anestesi Perioperatif, 5(2), 73. https://doi.org/10.15851/jap.v5n2.1106

[18] Soni, S. S., Cruz, D., Bobek, I., Chionh, C. Y., Nalesso, F., Lentini, P., De Cal, M., Corradi, V., Virzi, G., \& Ronco, C. (2010). NGAL: A biomarker of acute kidney injury and other systemic conditions. In International Urology and Nephrology (Vol. 42, Issue 1, pp. 141150). Springer. https://doi.org/10.1007/s11255009-9608-z
[19] Tejera D, Varela F, Acosta D, \& et al. (2017) Epidemiology of acute kidney injury and chronic kidney disease in the intensive care unit. Rev Bras Ter Intensiva, 29(4), 444-452.

[20] Vandenberghe, W., De Loor, J., \& Hoste, E. A. J. (2017). Diagnosis of cardiac surgery-associated acute kidney injury from functional to damage biomarkers. In Current Opinion in Anaesthesiology (Vol. 30, Issue 1, pp. 66-75). Lippincott Williams and Wilkins. https://doi.org/10.1097/AC0.0000000000000419

[21] Vives, M., Hernandez, A., Parramon, F., Estanyol, N., Pardina, B., Muñoz, A., Alvarez, P., \& Hernandez, C. (2019). Acute kidney injury after cardiac surgery: Prevalence, impact and management challenges. International Journal of Nephrology and Renovascular Disease, 12, 153-166.

https://doi.org/10.2147/IJNRD.S167477

[22] Wang, Y., \& Bellomo, R. (2017). Cardiac surgeryassociated acute kidney injury: Risk factors, pathophysiology and treatment. In Nature Reviews Nephrology (Vol. 13, Issue 11, pp. 697-711). Nature Publishing Group.

https://doi.org/10.1038/nrneph.2017.119

[23] Xiong T, Attar M, Gnirck AC, \& et al. (2020). Interleukin-9 protects from early podocyte injury and progressive glomerulosclerosis in Adriamycininduced nephropathy. Kidney International, 98(3), 615-629. 